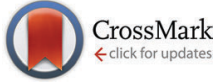

Cite this: Phys. Chem. Chem. Phys., $2015,17,21423$

Received 26th March 2015, Accepted 7th July 2015

DOI: $10.1039 / c 5 c p 01773 d$

www.rsc.org/pccp

\section{A facile one-pot synthesis of blue and red luminescent thiol stabilized gold nanoclusters: a thorough optical and microscopy study}

\begin{abstract}
Nabin Kumar Pal and Carola Kryschi*
Here in this contribution, blue and red luminescent 1-dodecanethiol (DT) terminated gold nanoclusters (AuNC) were prepared by a simple two-step synthesis route where the first step involved the surfactantfree synthesis of bare AuNC in N,N'-dimethylformamide (DMF) and the second step is the termination of the as-prepared bare AuNC by 1-dodecanethiol. The blue and red luminescent DT-terminated AuNC were isolated by a solvent-induced precipitation followed by an ultra-centrifugation technique. Both the bare AuNC and the blue and red luminescent DT-terminated AuNC exhibit stable photoluminescence and good solubility in various solvents. The photo-physical, electronic, structural, and morphological properties of the bare AuNC and the blue and red luminescent DT-terminated AuNC were examined by performing UV-Vis absorption spectroscopy, stationary and time-resolved PL spectroscopy, X-ray photoelectron spectroscopy (XPS), femtosecond transient absorption spectroscopy, Fourier-transform infrared spectroscopy (FTIR-ATR), and high-resolution transmission electron microscopy (HRTEM) experiments.
\end{abstract}

\section{Introduction}

Over the past two decades, few-atoms sub-nanometer sized, noble metal nanocluster have gained tremendous significance in both material and biological science. ${ }^{1-3}$ Owing to their existence, as a missing link between atoms and nanocrystals, these nanoclusters exhibit wide ranges of new material properties such as shape- and size-tunable optical, electronic, and electrical properties, molecular-like properties such as quantized charging, discrete energy states and size-dependent photoluminescence. ${ }^{4,5}$ These novel properties along with low toxicity and good biocompatibility make these nanocluster an important entity for researchers and make them feasible for applications in catalysis, ${ }^{6}$ optical sensing, ${ }^{7}$ bio-imaging, ${ }^{8}$ single moleculeoptoelectronics $^{9}$ and surface-enhanced Raman spectroscopy. ${ }^{10}$

The use of thiols as a protecting agent for the synthesis of gold nanoclusters is well established. The reason for this is mainly because of the formation of covalent bonds at the gold surface via sulphur head groups. The synthesis of thiol-derivatized stable gold nanoparticles was first reported by Brust and Schiffrin in the mid of $1990 .{ }^{11}$ Following this seminal work, various methods have been developed so far to synthesize monodisperse gold nanocluster of definite particle sizes by controlling the initial reaction conditions such as the thiol-to-gold ion ratio, reducing agents, temperature and also by modifying the post-synthesis

Department of Chemistry and Pharmacy and ICMM, Friedrich-Alexander-University of Erlangen-Nuremberg, Erlangen, Germany.E-mail: carola.kryschi@fau.de purification processes. Several well defined nanoclusters have been synthesized so far such as $\mathrm{Au}_{20}(\mathrm{SR})_{16}, \mathrm{Au}_{25}(\mathrm{SR})_{18}$, $\mathrm{Au}_{38}(\mathrm{SR})_{24}, \mathrm{Au}_{102}(\mathrm{SR})_{44}$, and $\mathrm{Au}_{144}(\mathrm{SR})_{60} \cdot{ }^{12-17}$ Among all these thiolate protected gold nanocluster, $\mathrm{Au}_{25}(\mathrm{SR})_{18}$ and $\mathrm{Au}_{144}(\mathrm{SR})_{60}$ are most widely studied due to their higher stability in comparison to other thiolated gold nanocluster. The X-ray crystal structure of the $\mathrm{Au}_{25}\left(\mathrm{SCH}_{2} \mathrm{CH}_{2} \mathrm{Ph}\right)_{18}$ nanocluster was also recently determined in both anionic and neutral forms. ${ }^{18}$ All these nanocluster were synthesized by using a thiol as a protecting ligand in an organic solvent. The use of organic solvents as a reaction medium makes these nanoclusters soluble only in organic solvents. However, the water solubility of the nanocluster is very crucial for biological applications (e.g. biological imaging, labelling and therapeutics). Several reports are available where gold nanoclusters were prepared using proteins, ${ }^{19}$ dendrimers,${ }^{20}$ amino acids, ${ }^{21} \mathrm{DNA}^{22}$ and polyelectrolytes $^{23}$ as scaffolds. However, most of the methods mentioned above need an expensive starting material, photoirradiation, electric field application or an external toxic reducing agent such as sodium borohydride. The synthesis of noble metal nanocluster without the use of external reducing agents was demonstrated in the recent past by Liz-Marzán and co-workers using $N, N^{\prime}$-dimethylformamide (DMF) as both a mild reducing and stabilizing agent. ${ }^{24}$ However, they only succeeded in producing non-luminescent nanoparticles with sizes larger than $5 \mathrm{~nm}$. These noble metal nanoparticles exhibit intense surface plasmon bands in the UV-Vis absorption spectrum. Later Liu et $a l^{25}$ extended this idea of using DMF as both a reducing and stabilizing agent at high temperature and they have succeeded in developing. 
a surfactant-free recipe to synthesize highly stable and luminescent gold nanocluster. Recently, Yabu et al. ${ }^{26}$ reported a novel one-pot process for the synthesis of AuNC via simple reduction of $\mathrm{Au}$ ions by amino-terminated poly(1,2-butadiene) $\left(\mathrm{PB}^{-\mathrm{NH}_{2}}\right)$ in toluene. The synthesized AuNC show PL-emission at $465 \mathrm{~nm}$ upon excitation at $370 \mathrm{~nm}$ and were characterized as $\mathrm{Au}_{8}$ clusters (from MALDI-TOF MS analysis).

In this contribution we refer to a surfactant-free synthesis route by preparing highly stable and blue luminescent AuNC using $N, N$-dimethylformamide (DMF) as both a reducing and protecting agent, following the method originally proposed by Liu et $a l^{25}$ with minor modifications. The as-synthesized bare AuNC were terminated with 1-dodecanethiol (1-DT). Moreover, here we demonstrate for the first time that the long-time aging reaction between a bare AuNC and an excess of 1-DT produces both blue and red luminescent DT-terminated AuNC in the same pot. These two differently sized nanoclusters were separated very conveniently through a solvent-induced precipitation method followed by ultracentrifugation. Both blue and red luminescent DT-terminated gold nanoclusters were readily dissolved in methanol and water for further characterization.

\section{Experimental parts}

\subsection{Materials}

Hydrogen tetrachloroaurate(III) trihydrate $\left(\mathrm{HAuCl}_{4} \cdot 3 \mathrm{H}_{2} \mathrm{O}, \geq 99.5 \%\right.$, p.a), $\mathrm{NaBH}_{4}(97 \%)$ and $N, N^{\prime}$-dimethylformamide ( $\left.\geq 99.8 \%\right)$ were purchased from Roth, and 1-dodecanethiol (DT, 99\%) and methanol (anhydrous, 99.8\%) were obtained from Aldrich. All materials and solvents were used as received from the suppliers without further purification. In all syntheses milli-Q de-ionised water (18 $\mathrm{M} \Omega$ ) was used.

\subsection{Synthesis of uncoated gold nanoclusters (AuNC)}

The bare AuNC were prepared according to the DMF reduction technique that was previously reported by Liu et $a .^{25}$ In a typical procedure, $30 \mathrm{ml}$ of $N, N^{\prime}$-dimethylformamide (DMF) was poured into a three neck round-bottom flask under an argon atmosphere. The flask was then slowly heated in a silicon-oil bath and kept as such until the temperature reaches $145{ }^{\circ} \mathrm{C}$. A solution of $300 \mu \mathrm{l}$ of $0.1 \mathrm{M}$ aqueous $\mathrm{HAuCl}_{4}$ was then added to this pre-heated DMF solution at once under vigorous stirring. The reaction was allowed to proceed for $6 \mathrm{~h}$ under reflux. After $6 \mathrm{~h}$ of refluxing a light yellow-coloured solution was obtained that was centrifuged thrice at $12000 \mathrm{rpm}$ for $30 \mathrm{~min}$, in order to remove the larger gold nanoparticles and other impurities. After centrifugation the solution was evaporated under high vacuum to remove the excess solvent. The residue thus obtained was dissolved in methanol for further characterization and functionalization.

\subsection{Functionalization of the as-prepared bare AuNC with 1-dodecanethiol (DT)}

To $3 \mathrm{ml}$ of the methanolic solution of the as-prepared AuNC, $0.1 \mathrm{mmol}$ of 1 -dodecanethiol (ligand-to-AuNC mole ratio $\approx 3$ ) was added under vigorous stirring. The reaction was allowed to proceed in the dark for about $24 \mathrm{~h}$. After the completion of the ligand binding process the solution was aged for another $24 \mathrm{~h}$. During this aging process some of the initially formed blue luminescent DT-terminated AuNC start to grow to larger red luminescent AuNC. Finally $5 \mathrm{ml}$ of de-ionised water was added which was followed by centrifugation at $12000 \mathrm{rpm}$ for $30 \mathrm{~min}$ to separate the blue and red luminescent DT-terminated AuNC. The red luminescent AuNC were obtained in the precipitate, whereas the blue luminescent AuNC remain in the solution. Both these DT-terminated AuNC were dissolved in methanol for further characterization.

\section{Methods and instrumentations}

\subsection{High-resolution transmission electron microscopy (HRTEM)}

The HRTEM images were recorded using a Phillips CM 300 UltraTwin microscope. The measurements were carried out at an accelerating voltage of $300 \mathrm{kV}$ in the bright-field mode. The samples for HRTEM were prepared by dropcasting 5 microliters of the ethanolic solution of the DT-terminated blue and red luminescent AuNC onto an ultrathin carbon coated copper grid (300 meshes) followed by drying in a vacuum at $25^{\circ} \mathrm{C}$ for 2 days.

\subsection{X-ray photoelectron spectroscopy}

The chemical compositions of the bare AuNC were investigated by X-ray photoelectron spectroscopy (XPS, PHI 5600 XPS spectrometer). Monochromatic $\mathrm{Al} \mathrm{K}_{\alpha}$ was used as the X-ray source.

\subsection{Fourier-transform infrared spectroscopy-attenuated total reflection (FTIR-ATR)}

The infrared spectrum was taken in the attenuated total reflection mode (ATR) using a FTIR-spectrometer (Prestige-21 Shimadzu) equipped with a smart-orbit ATR attachment containing a single reflection diamond crystal. The angle of incidence relative to the ATR crystal surface was $45^{\circ}$ in the analysis. Typically 100 scans were performed with a $4 \mathrm{~cm}^{-1}$ resolution.

\subsection{Steady-state optical spectroscopy}

The PL spectra were recorded on a Jobin-Yvon FluoroMax-3 spectrofluorometer using the magic-angle polarization configuration and a slit width of $5 \mathrm{~nm}$ for both excitation and emission spectra. The UV-Vis absorption spectra were recorded on a Perkin Elmer UV-Vis absorption spectrometer Lambda 2. All experiments were performed at room temperature using quartz cuvettes with an optical path length of $10 \mathrm{~mm}$. The PL quantum yields of the DT-terminated blue- and red-luminescent AuNC were estimated using a fluorescence standard. The following relative comparison equation was used to evaluate the quantum yield:

$$
\frac{Q_{\mathrm{t}}}{Q_{\mathrm{s}}}=\frac{\left(I_{\mathrm{t}} / A_{\mathrm{t}}\right) \eta_{\mathrm{t}}{ }^{2}}{\left(I_{\mathrm{s}} / A_{\mathrm{s}}\right) \eta_{\mathrm{s}}{ }^{2}}
$$

where $Q$ is the PL quantum yield, $I$ the integral area under the PL spectrum, $\eta$ the refractive index of the solvent, and $A$ the 
absorption at the selected excitation wavelength. The subscripts " $t$ " and "s" represent the test sample and the fluorescence standard, respectively.

\subsection{Time-resolved optical spectroscopy}

PL decay profiles were recorded using the time-correlated single-photon counting (TCSPC) PL spectroscopy technique. This time resolved measurement of the PL intensity was carried out on a TCSPC spectrometer Fluorolog-3 (Jobin Yvon) equipped with a microchannel plate (Hamamatsu, R3809U-50) that provides a time resolution of about $60 \mathrm{ps.}$

Femtosecond transient absorption spectroscopy experiments were conducted using a Clark MXR CPA 2101 laser system in conjunction with an Ultrafast TAPPS HELIOS detection system, consisting primarily of a glass-fiber based spectrometer. The output pulses at $387 \mathrm{~nm}$ with a $150 \mathrm{fs}$ pulse and a $1 \mathrm{kHz}$ repetition rate were used as pump pulses. They were obtained by amplifying and frequency doubling the $775 \mathrm{~nm}$ seeding pulses of the $\mathrm{Er}^{3+}$-doped glass fiber oscillator in a regenerative chirped-pulse titanium-sapphire amplifier and with the frequency doubling $\mathrm{BBO}$ crystal in the nonlinear optical amplifier (NOPA), respectively. All samples were pumped at excitation densities between $1.14 \times 10^{9}$ and $1.90 \times 10^{9} \mathrm{~W} \mathrm{~cm}^{-2}$. The samples consisting of $1 \mathrm{mg}$ of bare AuNC dispersed in $0.5 \mathrm{ml}$ of absolute ethanol were irradiated in quartz cuvettes with a thickness of $2 \mathrm{~mm}$. A fraction of the fundamental was simultaneously passed through a sapphire plate $(3 \mathrm{~mm})$ to generate the fs white-light continuum between 400 and $1400 \mathrm{~nm}$. The chirp between 400 and $750 \mathrm{~nm}$ was approximately $350 \mathrm{fs}$. Transient absorption spectra of the bare AuNC in ethanol were taken at delay times between $-0.5 \mathrm{ps}$ and $810 \mathrm{ps}$. They were recorded in the visible region between 420 and $750 \mathrm{~nm}$.

\section{Results and discussion}

\subsection{Synthesis of bare AuNC}

Here a simple one-pot synthesis method was employed to synthesize sub-nanometer sized AuNC. $N, N^{\prime}$-Dimethylformamide (DMF) was used as a solvent as well as a weak reducing agent. It is already known from other reports ${ }^{24,25}$ that DMF is able to act as a mild reducing agent at high temperature. In particular, the addition of a gold salt solution into a pre-heated DMF was observed here to perform as a redox reaction that predominantly yielded AuNC and a little or no formation of larger gold nanoparticles and bulk metals as by-products is observed.

The complete mechanism of the reduction reaction is not well understood. In many previous reports, ${ }^{24,25}$ it was believed that, during the process, dimethylformamide (DMF) oxidizes to carbamic acid which in turn at high temperature can easily decompose to dimethyl amine with the evolution of carbon dioxide gas.

$$
\begin{gathered}
\mathrm{HCONMe}_{2}+\mathrm{Au}(\mathrm{III})+\mathrm{H}_{2} \mathrm{O} \rightarrow 2 \mathrm{Au}^{0}+\mathrm{Me}_{2} \mathrm{NCOOH}+2 \mathrm{H}^{+} \\
\mathrm{Me}_{2} \mathrm{NCOOH} \rightarrow \mathrm{CO}_{2} \uparrow+\mathrm{Me}_{2} \mathrm{NH}
\end{gathered}
$$

The overall progress of the reduction reaction can be monitored by observing the colour change during heating. The initially yellow gold salt solution was changed to a colourless solution after half an hour of refluxing which is assumed to be due to the formation of $\mathrm{Au}(\mathrm{I})$ in the middle of the reduction process. Finally after $5 \mathrm{~h}$ of refluxing, a light brown solution was formed which remained unchanged on further heating implying the completion of the reduction process.

Further evidence for the formation of luminescent AuNC can be obtained from the blue luminescence which was observed to emerge from the as-synthesized AuNC under UV-light (365 nm) irradiation (Fig. 1). The AuNC dissolved in methanol exhibit a PL emission peak at $405 \mathrm{~nm}$ upon excitation at $330 \mathrm{~nm}$ (Fig. 2). The PL of such an AuNC is considered to arise from the electronic transitions between the $5 \mathrm{~d}^{10}$ valence band and the $6 \mathrm{sp}^{1}$ conduction band of the gold atom.

Fig. 3 shows the PL emission spectra of the AuNC that were synthesized at different refluxing times. Apparently the PL emission intensity increases with increasing refluxing time, and the intensity reaches a maximum after $5 \mathrm{~h}$ of refluxing. On further heating, almost no change in the PL emission intensity was observed which confirms the completion of the reduction reaction.

DMF can only act as a mild reducing agent at high temperature. In contrast, at low temperature, the same reaction will

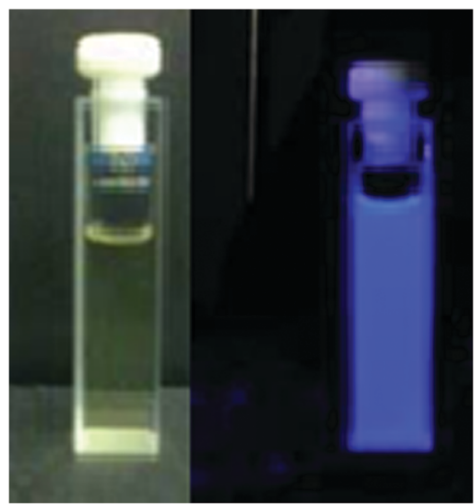

Fig. 1 Photographs of the as-synthesized bare AuNC under ambient light exposure (left) and under UV light irradiation at $364 \mathrm{~nm}$ (right).

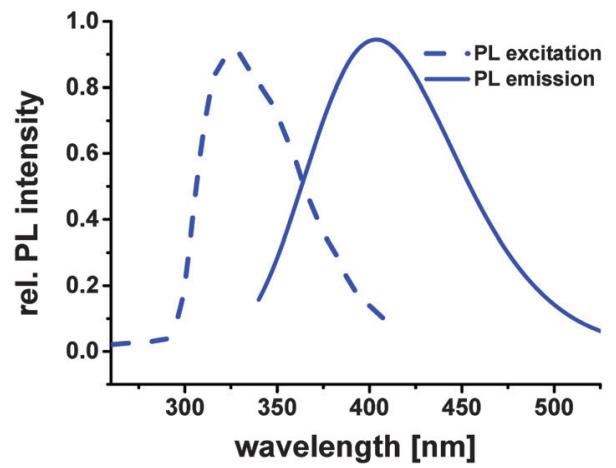

Fig. $2 \mathrm{PL}$ excitation (blue dashed line) and emission (blue solid line, excited at $330 \mathrm{~nm}$ ) spectra of bare AuNC. 
yield large gold nanoparticles that exhibit surface plasmon resonance absorption instead of PL emission. This implies that the formation of AuNC requires a sufficiently high temperature, in order to activate the reducing ability of DMF to reduce metallic gold ions to non-metallic luminescent AuNC. Fig. 4 shows the PL emission spectra of the products synthesized at three different refluxing temperatures that are $145{ }^{\circ} \mathrm{C}, 100{ }^{\circ} \mathrm{C}$ and $70{ }^{\circ} \mathrm{C}$, respectively. AuNC synthesized at $100{ }^{\circ} \mathrm{C}$ exhibit a very low-intensity PL emission, whereas the AuNC synthesized at $70{ }^{\circ} \mathrm{C}$ show no PL emission at all.

The UV-Vis absorption spectra (Fig. 5) of the as-synthesized AuNC do not contain any peak at $324 \mathrm{~nm}$ as being characteristic of $\mathrm{Au}$ (III) ions which suggests the complete reduction of $\mathrm{Au}$ (III) to $\mathrm{Au}(0)$. The absence of any surface plasmon resonance peak, which appears in the range of $520-570 \mathrm{~nm}$ for gold nanoparticles in the absorption spectra, indicates that the reaction predominantly produces AuNC with sizes less than $2 \mathrm{~nm}$. The absorption increases strongly towards shorter wavelengths at around $400 \mathrm{~nm}$ due to interband transitions. A broad absorption band can be observed at around 300-350 $\mathrm{nm}$ which is a finger-print band for few atoms sub-nanometer sized AuNC $\left(\mathrm{Au}_{4}-\mathrm{Au}_{10}\right)$. The absence of the plasmon absorption band in the case of sub-nanometer sized AuNC was previously explained in terms of the electron-surface scattering phenomenon. ${ }^{27,28}$ The absence of the localised surface plasmon resonance (LSPR)

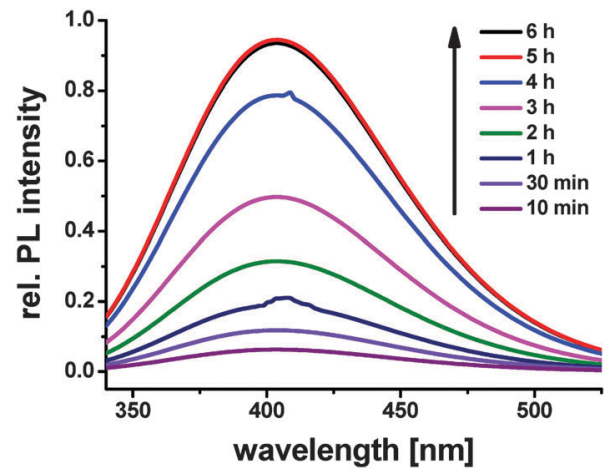

Fig. $3 \mathrm{PL}$ emission spectra of bare AuNC that were synthesized at different refluxing times (excited at $330 \mathrm{~nm}$ ).

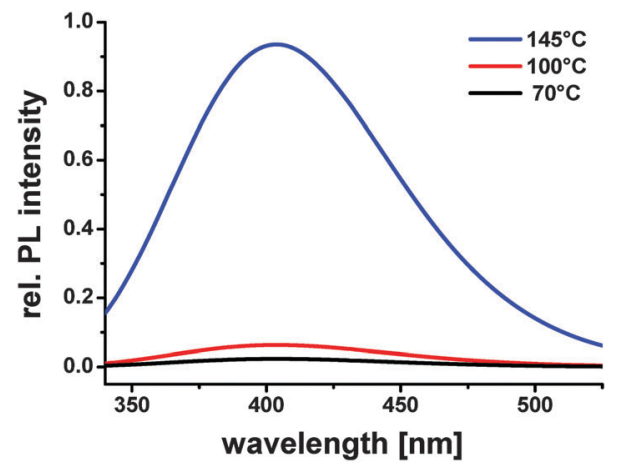

Fig. 4 PL emission spectra of bare AuNC synthesized at different refluxing temperatures: $145{ }^{\circ} \mathrm{C}$ (blue solid line), $100{ }^{\circ} \mathrm{C}$ (red solid line), and $70{ }^{\circ} \mathrm{C}$ (black solid line).

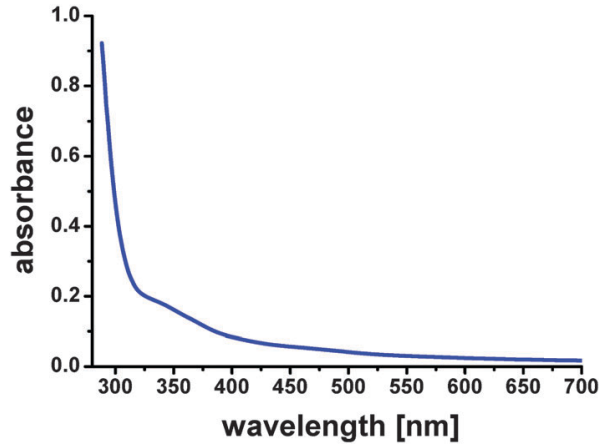

Fig. 5 The UV-Vis absorption spectrum of the as-synthesized bare AuNC.

band in small AuNC is also supported by the recent theoretical work on monolayer-protected AuNC carried out by Häkkinen and co-workers. ${ }^{29}$ In fact, they showed by performing ab initio calculations how the collective electronic oscillation may change when the gold core of the experimentally observed thiolate-stabilized gold cluster grows from 1.5 to $2.0 \mathrm{~nm}$. This theoretical study is of particular importance as it demonstrates a threshold size for the emergence of LSPR in these monolayerprotected AuNC. Almost no changes in the UV-Vis absorption spectra of the as-prepared bare AuNC were observed after 3 months of storage which indicates the great stability of the AuNC. Moreover, no alteration of the spectral features of their PL emission as well as no change in the UV-Vis absorption spectra were observed upon addition of a reducing agent such as $\mathrm{NaBH}_{4}$ or an oxidizing agent like $\mathrm{H}_{2} \mathrm{O}_{2}$. These experimental results prove both the high stability of the as-prepared AuNC and the absence of any Au ion in the solution.

The electronic structure and the oxidation state of the as-synthesized bare AuNC were examined upon performing XPS measurements. Fig. 6 shows the Au(4f) core-level photoemission spectrum of the freeze-dried AuNC. The $A u\left(4 \mathrm{f}_{7 / 2}\right)$ binding-energy peak of the resulting AuNC $(84.8 \mathrm{eV})$ is located between that of the $\mathrm{Au}(0)$ film $(84 \mathrm{eV})$ and those of the $\mathrm{Au}(\mathrm{I})$ species $(86.0 \mathrm{eV})$. This result further indicates that the reaction product mainly consists of AuNC. The dependence of the Au-4f binding energy on the nature of the surrounding ligands was previously reported. ${ }^{30-32} \mathrm{~A}$ detailed peak-shape analysis of thiolate-passivated $\mathrm{Au}$ nanoparticles carried out by Tanaka and co-workers showed that $\mathrm{Au}\left(4 \mathrm{f}_{7 / 2}\right)$ peaks are indeed composed of two components, one with lower binding energy originating from inner $\mathrm{Au}$ atoms and the other with relatively high binding energy arising from surface Au atoms linked to thiol molecules. ${ }^{30}$ The $4 \mathrm{f}_{7 / 2}$ binding energy of the surface $\mathrm{Au}$ atoms is generally higher in energy, since electron donation from surface Au atoms to the thiolate ligand occurs (initial-state effect). In the present study, the $\operatorname{Au}\left(4 \mathrm{f}_{7 / 2}\right)$ core level binding energy in the cluster is slightly higher than the $\mathrm{Au}\left(4 \mathrm{f}_{7 / 2}\right)$ binding energy value in an $\mathrm{Au}(0)$ film. This increase of the binding energy value is due to the small size of the cluster, a phenomenon explained in terms of the "finalstate effect", where a coulombic attractive interaction with the photocreated holes lowers the kinetic energy of the outgoing photoelectrons. 


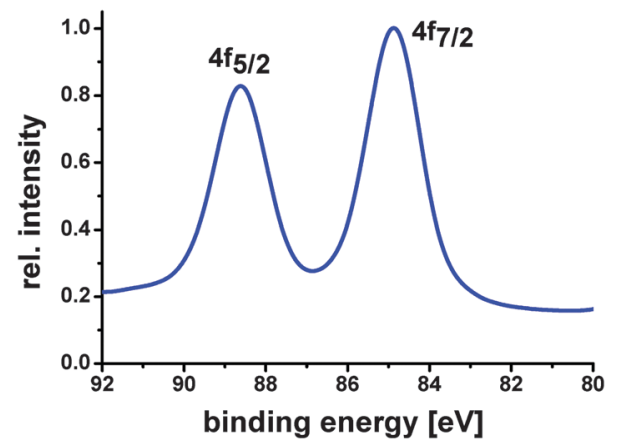

Fig. 6 Au(4f) XPS spectrum of the bare AuNC.

The PL emission lifetime measurements of the as-synthesized AuNC were performed by detecting their PL emission at $405 \mathrm{~nm}$ upon laser excitation at $295 \mathrm{~nm}$. The PL emission decay curve was simulated by employing a convolution of the apparatus response and a bi-exponential decay function $f(t)$ with $f(t)=$ $a_{1} \times \exp \left(-t / \tau_{1}\right)+a_{2} \times \exp \left(-t / \tau_{2}\right)$ (Fig. 7). The predominant long lifetime $3.2 \mathrm{~ns}(65 \%)$ can be associated with the PL emission of the small sized AuNC, whereas the shorter decay time $0.8 \mathrm{~ns}$ $(35 \%)$ is assigned to charge-transfer states. Obviously, the AuNC exhibit PL emission that decays on the ns time scale, which is in contrast to non-emitting AuNPs. ${ }^{33}$

The as-synthesized bare AuNC were further examined by HRTEM. Unfortunately no nanocluster with regular shapes were resolved. This observation is explained with the subnanometer size of the AuNC prepared in the absence of any stabilizing ligands and the limitation of lateral resolution of the used TEM. This finding is in good agreement with formerly published results. ${ }^{34,35}$

Using fs transient absorption spectroscopy the ultrafast photo-induced electron transfer and excited state dynamics of the AuNC were investigated. The transient absorption of the AuNPs and AuNC was previously studied..$^{36-38}$ The time evolution of the transient absorption spectra of the excited-state relaxation dynamics of bare AuNC following an excitation at $387 \mathrm{~nm}$ is depicted in Fig. 8. The excitation energy density and the time resolution used for the measurement were $1.5 \times 10^{9} \mathrm{~W} \mathrm{~cm}^{-2}$ and

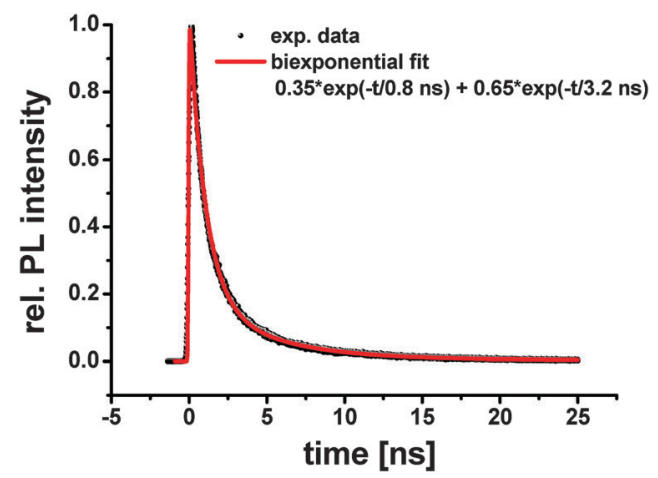

Fig. 7 The PL emission decay of the bare AuNC detected at $405 \mathrm{~nm}$ and excited at $295 \mathrm{~nm}$. The black dots are the experimental data, whereas the solid line (red) is the best fit.

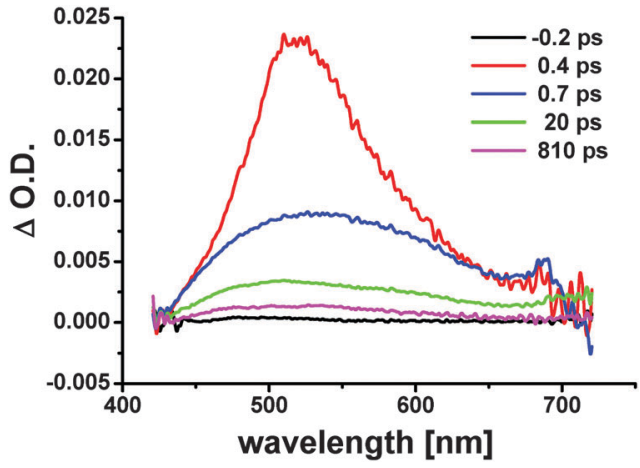

Fig. 8 Temporal evolution of the fs transient absorption spectra of the bare AuNC.

$180 \mathrm{fs}$, respectively. The spectra exhibit a broad photo-induced excited state absorption signature between $400 \mathrm{~nm}$ and $700 \mathrm{~nm}$. The observed positive transient absorption peak at around $510 \mathrm{~nm}$ is in sharp contrast to the transient absorption spectra of larger gold nanoparticles, where the transient absorption spectra represent the superposition of a broad absorption interband component with the bleach due to plasmon resonance.

Fig. 9 depicts a representative kinetic trace (detected at $530 \mathrm{~nm}$ ) which reflects the temporal behaviour of all kinetic traces monitored between 400 and $700 \mathrm{~nm}$. The kinetic traces were analyzed by performing simulations with a bi-exponential function that is deconvoluted with a 240 fs Gaussian function representing the apparatus response. The kinetic traces are unambiguously characteristic of a molecule-like cluster. In all kinetic traces, the bi-exponential decay dynamics comprises a fast (sub-picoseconds) and a slower (picoseconds) component. The sub-picosecond decay process presumably arose from electron-electron scattering, whereas the picosecond relaxation dynamics (4.1 ps) may be ascribed to radiationless processes such as internal conversion (IC) or intersystem crossing (ISC) which involve either promoting-mode coupling between the electronic ground and excited states or spin-orbit coupling among electronic states with different spin multiplicities.

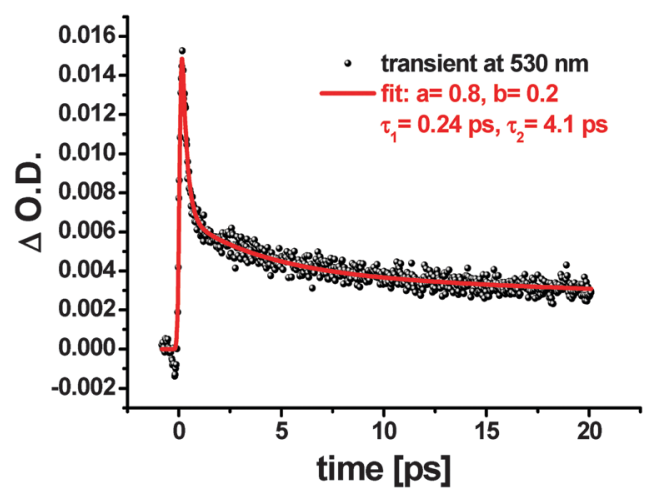

Fig. 9 Short time behaviour of the kinetic trace detected at $530 \mathrm{~nm}$. The black dots are the experimental data, whereas the solid line (red) is the best fit. 


\subsection{Termination of the bare AuNC}

The bare AuNC were terminated with 1-dodecanethiol (DT). Since 1-dodecanethiol contains an end-standing thiol group, this ligand binds via a covalent $\mathrm{S}-\mathrm{Au}$ bond. The covalent ligand binding was performed by stirring a methanolic solution of the bare AuNC with 1-dodecanethiol in the dark. After approximately $24 \mathrm{~h}$ of stirring, the thiol-terminated AuNC were aged for another $24 \mathrm{~h}$. Due to their small sizes AuNC have a high diffusion velocity in liquids so that inter-cluster atomic exchange processes may occur. This implies that aging processes in solutions such as Ostwald ripening may result in changes in the cluster size distribution. The observed changes in the average cluster size, surface morphology and optical properties can be monitored by optical and microscopy techniques. Here we observed that during the aging process DT-terminated AuNC grew to larger AuNC which altered the optical properties. A solvent-selective precipitation followed by ultra-centrifugation was employed to separate the larger nanocluster from the smaller ones in solution. The AuNC in the precipitate exhibited very intense red PL emission, while the rest of the solution showed blue PL under UV-light (365 nm) irradiation (Fig. 10).

The blue-luminescent DT-terminated AuNC exhibited a PL emission maximum at around $412 \mathrm{~nm}$ when excited at $330 \mathrm{~nm}$, whereas the red-luminescent DT-terminated AuNC showed a PL emission peak at 665 upon excitation at $370 \mathrm{~nm}$ (Fig. 11). The PL emission maximum of the bare AuNC is slightly red shifted (from $405 \mathrm{~nm}$ to $412 \mathrm{~nm}$ ) upon ligand attachment. The PL of the AuNC is assumed to originate from the electronic transition between the filled $5 \mathrm{~d}$ band and the 6 sp conduction band of the gold atom. As the size of the AuNC increases, the energy gap between the discrete states in each band decreases. This leads to a red-shift of the PL emission relative to that emerging from smaller AuNC. The PL quantum yield of the blue- and redluminescent DT-terminated AuNC was determined using quinine sulphate and rhodamine $\mathrm{B}$ as the fluorescence standard, and the obtained values are $8.2 \%$ and $6.7 \%$ for the blue- and red-luminescent DT-terminated AuNC, respectively. Both the

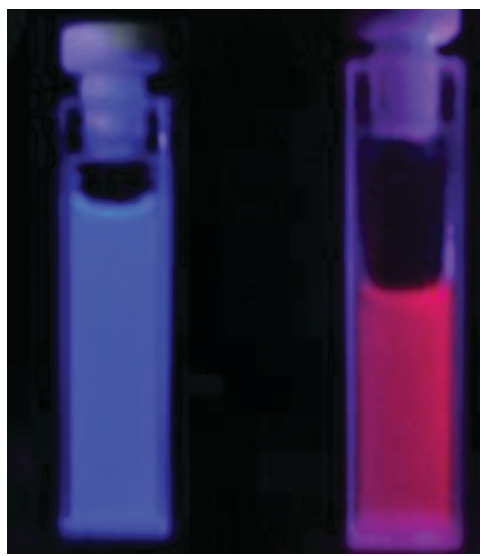

Fig. 10 Photographs of blue (left) and red luminescent (right) DTterminated AuNC under UV (365 nm) light exposure.

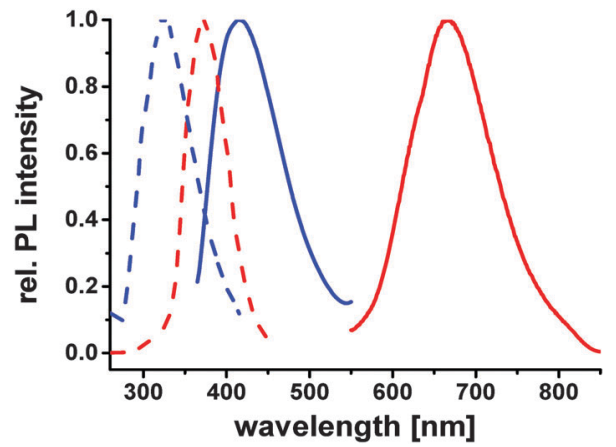

Fig. $11 \mathrm{PL}$ excitation spectra of the blue (blue dashed line) and red (red dashed line) luminescent DT-terminated AuNC, whereas the PL emission spectra of the blue and red luminescent DT-terminated AuNC are drawn with blue and red solid lines, respectively.

blue- and red-luminescent DT-terminated AuNC show great chemical stability in acidic and basic media. To examine the colloidal stability of these clusters, their PL emission spectrum was recorded in solution at different $\mathrm{pH}$ values (Fig. 12). Almost the same PL intensity of the blue and red DT-terminated AuNC was observed under all solvent conditions suggesting the high chemical stability of the DT-terminated AuNC in acidic and basic environments.

To further confirm the ligand binding to the gold core, FTIR measurements of the DT-terminated AuNC before aging were performed in the attenuated total reflection mode (ATR mode). The FTIR spectra of the DT-terminated AuNC and pure DT are presented in Fig. 13. Most of the characteristic vibrational
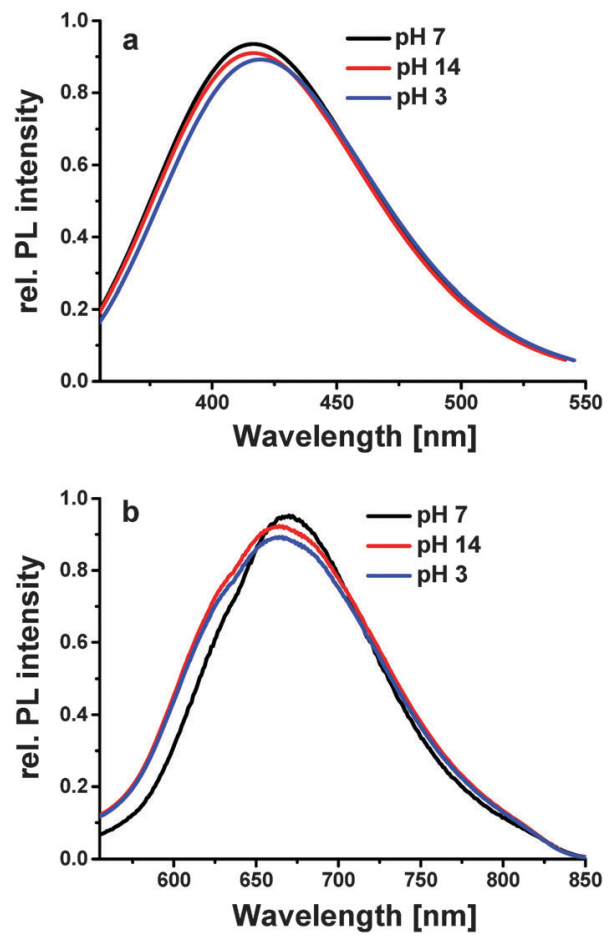

Fig. $12 \mathrm{PL}$ emission spectra of blue (a) and red luminescent (b) DTterminated AuNC in solution at $\mathrm{pH}$ values of 3,7 , and 14 , respectively. 
bands of the DT-terminated AuNC resemble those of pure DT which proves that DT is indeed a part of the cluster. Both the DT-terminated AuNC and pure DT exhibit sharp bands at $2923 \mathrm{~cm}^{-1}$ and $2850 \mathrm{~cm}^{-1}$ which can be assigned to the asymmetric and symmetric methylene stretching modes, respectively. Two bands at $2954 \mathrm{~cm}^{-1}$ and $2872 \mathrm{~cm}^{-1}$ are also present in the spectrum of the DT-terminated AuNC. These vibrational peaks are attributed to the asymmetric and symmetric stretching modes of the methyl group, respectively. The vibrational peaks in the range of $1000-1500 \mathrm{~cm}^{-1}$ are assigned

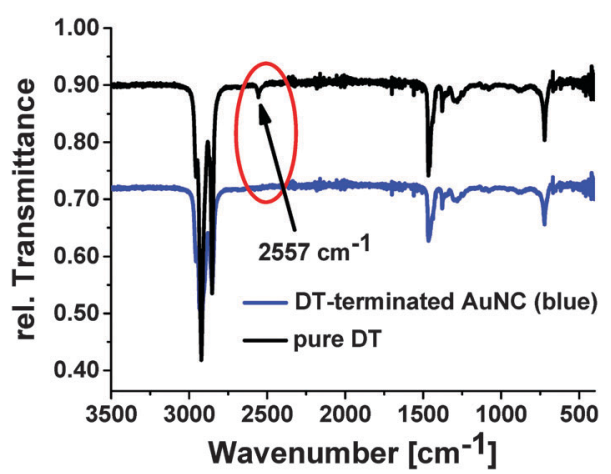

Fig. 13 FTIR-ATR spectra of pure DT (black solid line) and the blue luminescent DT-terminated AuNC (blue solid line). to the $\mathrm{C}-\mathrm{H}$ bending, wagging and $\mathrm{C}-\mathrm{C}$ stretching vibration modes. The bands at $663 \mathrm{~cm}^{-1}$ and $722 \mathrm{~cm}^{-1}$ in the FTIR spectrum are attributed to the $\mathrm{C}-\mathrm{S}$ stretching vibration of the thiol ligand. The pure 1-DT exhibits S-H stretching vibrational modes appearing at around $2557 \mathrm{~cm}^{-1}$. As expected, subsequent to its reaction with bare AuNC the S-H bond stretching vibrational band completely disappears which indicates the formation of the S-Au bond. The absence of the S-H stretching vibration peak is marked by a red circle (see Fig. 13).

In general, bare AuNC without any surface passivated ligands are too small to be visible in a TEM. However, upon surface stabilization the sizes of the AuNC increase a bit, so that they become visible under a high-resolution transmission electron microscope (HRTEM) recorded at an accelerating voltage of $300 \mathrm{kV}$. The HRTEM images of the blue- and red-luminescent DT-terminated AuNC are depicted in Fig. 14. The blue-luminescent DT-terminated AuNC are smaller than $1 \mathrm{~nm}$ and cannot be laterally resolved. Some of the nanoclusters are marked by arrows in the HRTEM image (Fig. 14C). However, the red-luminescent DT-terminated AuNC were isolated on the grid with a definite spherical shape. These red-luminescent DT-terminated AuNC show high monodispersity and the average size of these spherical particles was estimated to be $1.3 \pm 0.2 \mathrm{~nm}$.

Similar to bare AuNC, the time evolution of the PL emission for blue- and red-luminescent DT-terminated AuNC also includes two
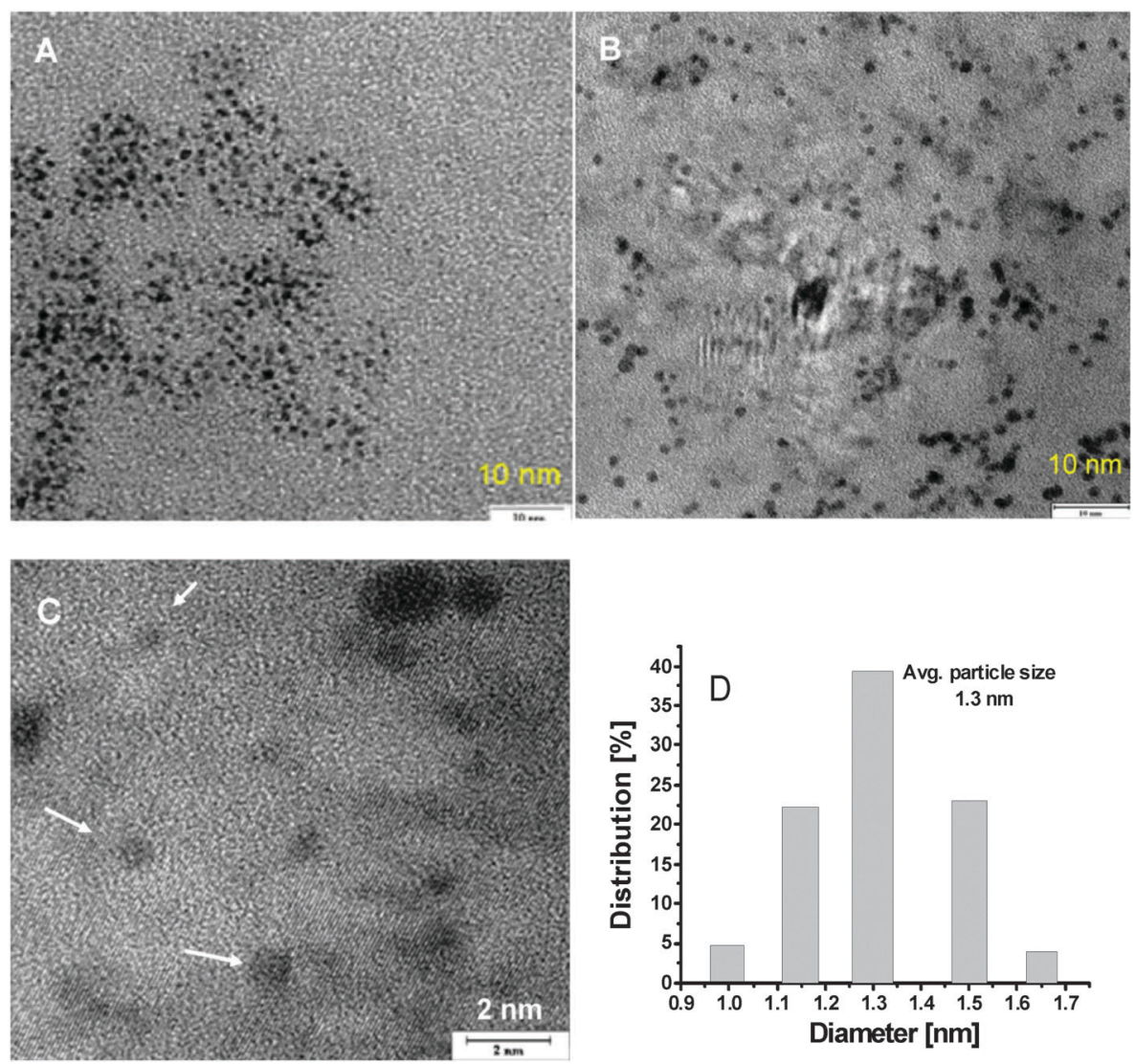

Fig. 14 HRTEM images of the blue (A and C) and red luminescent (B) DT-terminated AuNC. A histogram of the size distribution of the red-luminescent DT-terminated AuNC is shown in (D). 

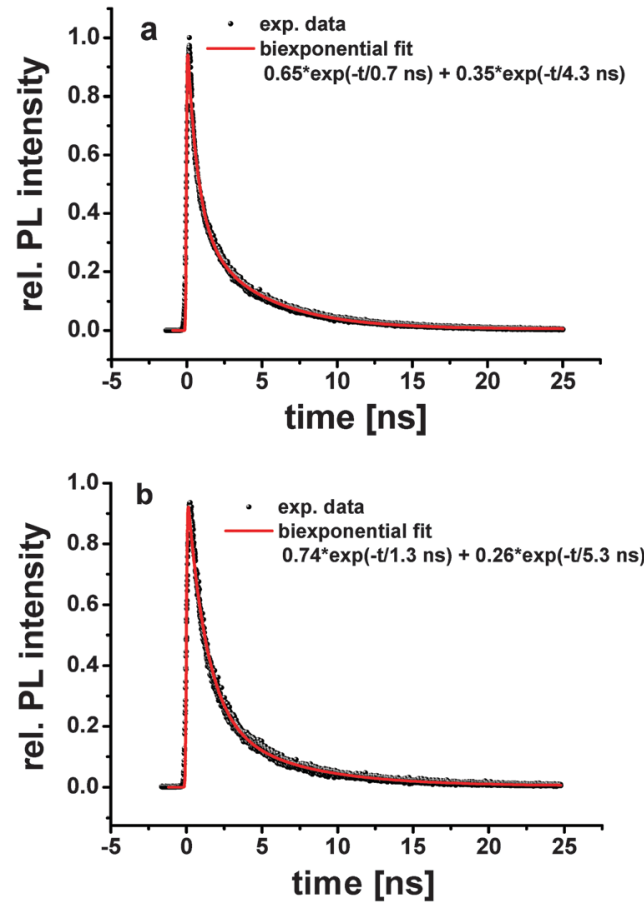

Fig. 15 PL emission decay profile of the blue (a: detected at $420 \mathrm{~nm}$ ) and red luminescent (b: detected at $660 \mathrm{~nm}$ ) DT-terminated AuNC (the black dots are the experimental data whereas the solid red line is the best fit).

exponential components as shown in Fig. 15. The fast and slow components have lifetimes of $0.7 \mathrm{~ns}(39 \%)$ and $4.3 \mathrm{~ns}(61 \%)$ for the blue-luminescent DT-terminated AuNC whereas the redluminescent DT-terminated AuNC have lifetimes of $1.25 \mathrm{~ns}$ (33\%) and 5.3 ns (67\%), respectively. The observed longer lifetimes of both kinds of AuNC are in good agreement with already published results ${ }^{27,28}$ and can be attributed to singlet transitions between low-lying $\mathrm{d}$ bands and excited sp bands.

\section{Conclusions}

In summary, we explored the mild reducing ability of $N, N^{\prime}$-dimethylformamide (DMF) for the synthesis of luminescent AuNC. The synthesis does not require any external surfactant or protecting ligands. The AuNC are very small in size $(<1 \mathrm{~nm})$ and show molecule-like features in their UV-Vis absorption spectra. The PL lifetime was found to be on the ns time scale. In addition, the fs transient absorption spectroscopy study indicates the presence of two excited-state lifetimes among which the fast sub-picosecond relaxation times at all wavelengths are assumed to originate from electron-electron scattering and the picosecond lifetimes are attributed to originate either from internal conversion or from intersystem crossing. In order to stabilize bare AuNC, 1-dodecanethiol (DT) was used as a protecting ligand. The ligand conjugation with the cluster was confirmed by FTIR spectroscopy. Moreover, the DT-terminated AuNC on further long time aging were observed to grow to larger-sized AuNC. The larger DT-terminated AuNC can be easily separated from the smaller ones employing a solvent-induced precipitation followed by the ultra-centrifugation technique. Smaller DT-terminated AuNC with sizes smaller than $1 \mathrm{~nm}$ show blue PL emission, whereas the larger DT-terminated AuNC with an average size of about $1.3 \mathrm{~nm}$ exhibit prominent red PL emission. Both the blue and red DT-terminated AuNC exhibit PL lifetimes on the ns time scale.

\section{Acknowledgements}

Support from the Deutsche Forschungsgemeinschaft (GRK 1161/2) is gratefully acknowledged.

\section{References}

1 L. Li, Q. Guo, J. Li, W. Yan, C. Leng, H. Tang, Q. Lu and B. Tan, J. Mater. Chem. B, 2013, 1, 3999.

2 J. Zheng, P. R. Nicovich and R. M. Dickson, Annu. Rev. Phys. Chem., 2007, 58, 409.

$3 \mathrm{X} . \mathrm{Wu}, \mathrm{X} . \mathrm{He}, \mathrm{K} . \mathrm{Wang}, \mathrm{C} . \mathrm{Xie}, \mathrm{B}$. Zhou and Z. Qing, Nanoscale, 2010, 2, 2244.

4 C. B. Murray, D. J. Norris and M. G. Bawendi, J. Am. Chem. Soc., 1993, 115, 8706.

5 W. C. W. Chan and S. Nie, Science, 1998, 281, 2016.

6 H. Tsunoyama, N. Ichikuni, H. Sakurai and T. Tsukuda, J. Am. Chem. Soc., 2009, 131, 70.

7 T. Zhou, M. Rong, Z. Cai, C. James Yang and X. Chen, Nanoscale, 2012, 4, 4103.

8 N. Makarava, A. Parfenov and I. V. Baskakov, Biophys. J., 2005, 89, 572.

9 T. H. Lee, J. I. Gonzalez, J. Zheng and R. M. Dickson, Acc. Chem. Res., 2005, 38, 534.

10 M. Scolari, A. Mews, N. Fu, A. Myalitsin, T. Assmus, K. Balasubramanian, M. Burghard and K. Kern, J. Phys. Chem. C, 2008, 112, 391.

11 M. Brust, M. Walker, D. Bethell, D. J. Schiffrin and R. Whyman, J. Chem. Soc., Chem. Commun., 1994, 801-802.

12 Y. Shichibu, Y. Negishi, T. Tsukuda and T. J. Teranishi, J. Am. Chem. Soc., 2005, $127(39), 13464-13465$.

13 Z. Wu, J. Suhan and R. J. Jin, Mater. Chem., 2009, 19, 622.

14 T. G. Schaaff and R. L. Whetten, J. Phys. Chem. B, 2000, 104, 2630.

15 H. Qian, M. Zhu, U. N. Andersen and R. J. Jin, J. Phys. Chem. A, 2009, 113(16), 4281-4284.

16 Y. Levi-Kalisman, P. D. Jadzinsky, N. Kalisman, H. Tsunoyama, T. Tsukuda, D. A. Bushnell and R. D. Kornberg, J. Am. Chem. Soc., 2011, 133(9), 2976-2982.

17 H. Qian. R. Jin, Chem. Mater., 2011, 23(8), 2209-2217.

18 T. Dainese; S. Antonello, J. A. Gascon, F. Pan, N. V. Perera, M. Ruzzi, A. Venzo, A. Zoleo; K. Rissanen and F. Maran, ACS Nano, 2014, 8(4), 3904-3912.

19 N. K Pal and C. Kryschi, Phys. Chem. Chem. Phys., 2015, 17, 1957.

20 J. Zheng and R. M. Dickson, J. Am. Chem. Soc., 2002, 124, 13982. 
21 N. K Pal and C. Kryschi, J. Mol. Catal. A: Chem., 2015, 404, 27-35.

22 J. T. Petty, J. Zheng, N. V. Hud and R. M. Dickson, J. Am. Chem. Soc., 2004, 126, 5207.

23 L. Shang and S. J. Dong, Chem. Commun., 2008, 1088.

24 I. Pastoriza-Santos and L. M. Liz-Marzán, Langmuir, 1999, 15, 948.

25 X. Liu, C. Li, J. Xu, J. Lu, M. Zhu, Y. Guo, S. Cui, H. Liu, S. Wang and Y. Li, J. Phys. Chem. C, 2008, 112, 10778.

26 H. Yabu, Chem. Commun., 2011, 47, 1196-1197.

27 U. Kreibig and C. von Fragstein, Z. Phys., 1969, 224, 307.

28 U. Kreibig, Z. Phys., 1970, 234, 307.

29 S. Malola, L. Lehtovaara, J. Enkovaara and H. Häkkinen, ACS Nano, 2013, 7(11), 10263-10270.

30 A. Tanaka, Y. Takeda, M. Imamura and S. Sato, Phys. Rev. B: Condens. Matter Mater. Phys., 2003, 68, 195415.
31 H. Grönbeck and M. Odelius, Phys. Rev. B: Condens. Matter Mater. Phys., 2010, 82, 085416.

32 A. Chaudhuri, T. J. Lerotholi, D. C. Jackson and D. P. Woodruff, Phys. Rev. Lett., 2009, 102, 126101.

33 K. V. Mrudula, T. Udaya Bhaskara Rao and T. Pradeep, J. Mater. Chem., 2009, 19, 4335.

34 H. Duan and S. Nie, J. Am. Chem. Soc., 2007, 129, 2412-2413.

35 R. Jin, S. Egusa and N. F. Scherer, J. Am. Chem. Soc., 2004, 126, 9900-9901.

36 M. Farrag, M. Thämer, M. Tschurl, T. Bürgi and U. Heiz, J. Phys. Chem. C, 2012, 116, 8034.

37 C. Zhou, C. Sun, M. Yu, Y. Qin, J. Wang, M. Kim and J. Zheng, J. Phys. Chem. C, 2010, 114, 7727.

38 Y. Hong, J. W. Y. Lam and B. Z. Tang, Chem. Soc. Rev., 2011, 40, 5361 . 